progenitor cells in Matrigel were applied to the adventitia of the injured femoral artery, REP+ cells were observed in the intimal 24 hours post-operation, which significantly enhanced neointimal lesions at 2 weeks. This increased neointimal lesion was rescued by pre-treatment of $\mathrm{Sca}-1+$ cells with leptin antagonist CYT-566. In addition, when wild-type Sca-1+ progenitor cells were delivered to the adventitia of the injured artery in leptin receptor deficient mice, a significant increased neointimal formation was observed at 2 weeks, indicating crucial roles of leptin receptor and Sca-1+ progenitor cells in vascular remodelling.

Conclusions- Levels of leptin in both the vessel wall and the circulation are upregulated after vessel injury, leading to the migration of Sca-1+ progenitor cells that enhances neointimal formation.

\section{CARDIOPROTECTIVE ROLE OF FINGOLIMOD IN ISCHEMIA-REPERFUSION INJURY BY ACTIVATION OF AKT/ERK PATHWAYS IN RAT MODEL}

${ }^{1}$ Naseer Ahmed*, Daniele Linardi, Sajeela Ahmed, ${ }^{2}$ Riffat Mehboob, Giovanni B Luciani, ${ }^{1}$ Giuseppe Faggian, ${ }^{1}$ Alessio Rungatscher. ' University of Verona; ${ }^{2}$ King Edward Medical University, Biomedical Sciences

\subsection{6/heartjnl-2017-311726.166}

Background and purpose Ischaemic Heart Diseases (IHD) are the most common cause of morbidity and mortality. Incidence and prevalence are continuously growing. There is an escalating risk for revascularisation or resuscitation in patients with IHD. Recently, it has been reported that a sphingosine 1-phosphate receptor agonist play an anti-apoptotic and anti-inflammatory role in the ischemia-reperfusion injury.

Objectives The aim of our study is to investigate the cardioprotective effects of sphingosine 1-phosphate receptor agonist fingolimod (FTY720) on global ischemia-reperfusion injury related to the cardiac surgery.

Methods In our experimental study, global ischemia-reperfusion was achieved by cardiopulmonary bypass by cardioplegic arrest on ventilated male Sprague-Dawley rats (300-350 g). The global ischaemic period lasted $10 \mathrm{~min}$ in the cardioplegic arrest while reperfusion times were maintained for $60 \mathrm{~min}$ and 24 hours. ECG monitoring was done using AD instrument and using Millar catheter, heart rate, systolic and diastolic pressures were recorded and mean arterial pressure was calculated. The statistical significance was considered at P 0.05 .

Results The myocardial protection was observed in the group treated with Fingolimod as compared to control groups. Reduced frequency of apoptotic cells and inflammatory mediators were found in the treated group. The level of adenylates was preserved in the treated group as compared to controls (94\%, 61\% respectively)(p0.001). Reactive Oxygen Species (ROS) were attenuated in the fingolimod-treated group. Fingolimod treatment improved systolic and diastolic ventricular pressures and contractility strength (p0.005).

Conclusions The intravenous administration of fingolimod in global ischemia-reperfusion was cardioprotective. Fingolimod cardioprotection appears to be mediated through preservation of high energy phosphates, reduction in oxidative stress, inhibition of apoptosis and inflammation leading to improved cardiac functions.

\section{9} DEVELOPING CAPTURE ASSAYS TO MEASURE CIRCULATING CRP/OXIDISED LOW DENSITY LIPOPROTEIN COMPLEXES IN PATIENTS UNDERGOING CARDIOPULMONARY BYPASS AND EXAMINING USE OF CRP/OXIDISED LOW DENSITY LIPOPROTEIN COMPLEXES AS A BIOMARKER OF ATHEROSCLEROSIS

${ }^{1}$ Prachi Mann*, ${ }^{2}$ Ramzi YJ Khamis, ${ }^{2}$ Dorian Haskard²Minh Ta, ${ }^{3}$ Chiari Kojima ${ }^{2}$ Tareq Ammari, ${ }^{3}$ Mikhail Caga-Anan, ${ }^{4}$ Bao A Nguyen, ${ }^{52}$ Jonathan R Anderson, ${ }^{6}$ Paul C Evans, ${ }^{3}$ Michael Johns. 'Brighton and Sussex Medical School; ${ }^{2}$ Imperial College London; ${ }^{3}$ National Heart \& Lung Institute, Imperial College London; ${ }^{4}$ University of Bristol; ${ }^{5}$ Imperial College Healthcare NHS Trust; ${ }^{6}$ Department of Infection, Immunity \& Cardiovascular Disease, University of Sheffield

\subsection{6/heartjnl-2017-311726.167}

Background Atherosclerosis is a disease of global importance. It is a systemic chronic inflammatory disease with a complex aetiology. LDL is primarily oxidised in the subendothelial space, as plasma antioxidants protect circulating LDL from oxidation. However, small amounts of oxidised LDL (oxLDL) have been detected in plasma. Modified LDL is thought to circulate as part of an immune complex; these immune complexes may also include CRP, complement and IgM antibodies. This project aims to develop a capture ELISA to measure oxLDL/CRP complexes in circulation, to help better characterise circulating immune complexes and explore potential use of oxLDL/CRP complexes a biomarker of atherosclerosis.

Methods A CRP/oxLDL capture ELISA was developed using LO1, a novel mAb specific to oxLDL. Plasma samples were obtained from patients undergoing cardiopulmonary bypass at four different timepoints. Levels of oxLDL, CRP and oxLDL/ CRP complexes were determined in patient plasma using capture assays developed during this study.

Results OxLDL levels show an uptrend from baseline to $60 \mathrm{mins}$, followed by significant reduction at $300 \mathrm{mins}$ compared to baseline $(0.506 \pm 0.078$ vs $0.456 \pm 0.035 ; p=0.0138)$. CRP concentration initially declines from baseline up to $120 \mathrm{mins}(1.918 \pm 0.915$ vs $1.136 \pm 0.599 ; p=0.0009)$, after which levels rise between $120 \mathrm{~min}$ and $300 \mathrm{~min}(1.136 \pm 0.599$ vs $1.782 \pm 0.65 ; \mathrm{p}=0.0026)$. There is no significant difference in levels of CRP/oxLDL complexes across all timepoints.

Conclusion It is possible to measure CRP/oxLDL complexes in plasma. Pro-inflammatory events such as cardiopulmonary bypass are associated with changes in levels of oxLDL and CRP, while oxLDL/CRP levels remain unchanged.

\section{FETUIN-A INDUCES NOX1-ROS-DEPENDENT VASCULAR DYSFUNCTION PARTIALLY THROUGH TOLL LIKE RECEPTOR 4 ACTIVATION}

Augusto Montezano*, Rheure Lopes, Karla Neves, Delyth Graham, Rhian M Touyz. University of Glasgow

\subsection{6/heartjnl-2017-311726.168}

Fetuin-A (FetA) is a plasma glycoprotein important for the regulation of calcium and phosphate homeostasis. It is also an agonist to toll-like receptor 4 (TLR4) and is related to insulin resistance and inflammation. FetA has also been associated with endothelial dysfunction, which is regulated by oxidative stress. Mechanisms whereby FetA influences vascular function are unknown. We hypothesised that FetA through TLR4 and ROS production induces vascular dysfunction. Mesenteric 
arteries and vascular cells from WKY rats were studied. Vascular function was analysed by wire myography in the presence or absence of FetA $(50 \mathrm{ng} / \mathrm{mL})$ and/or CLI095 (CLI $-10^{-6} \mathrm{M}$ - TLR4 inhibitor). Reactive oxygen species (ROS) were measured by chemiluminescence, Amplex Red $\left(\mathrm{H}_{2} \mathrm{O}_{2}\right)$ and ELISA to nitrotyrosine levels (peroxynitrite $-\mathrm{ONOO}^{-}$). Protein oxidation and levels were measured by immunoblotting. WKY vessels exposed to FetA were less sensitive to acetylcholine (Ach)-induced and sodium nitroprusside (SNP)-induced relaxation, while sensitivity to phenylephrine was increased by FetA; an effect blocked by N-acetylcysteine (antioxidant) and ML171 (Nox1 inhibitor). Inhibition of TLR4 blocked FetA effects on endothelial-dependent relaxation and contraction, but not on endothelial-independent relaxation. FetA increased ROS production ( 1 fold), but decreased $\mathrm{H}_{2} \mathrm{O}_{2}$ intracellular levels $(0.5$ fold); and increased gene levels of IL6 (2 fold), IL1 $\tilde{A} \square \hat{A}^{2}$ (1 fold), RANTES (1 fold) and MMP2/9 (2 fold) in endothelial cells (EC) $(\mathrm{p}<0.05)$; an effect blocked by CLI095. ROS production (0.5 fold), as well as, $\mathrm{H}_{2} \mathrm{O}_{2}$ (0.5 fold) and $\mathrm{ONOO}^{-}$(1 fold) levels, were increased by FetA in VSMCs $(\mathrm{p}<0.05)$. Protein oxidation was also increased by FetA in VSMCs (1 fold, $\mathrm{p}<0.05)$. In EC, eNOS inactivation (1 fold) and JNK activation $(0.5$ fold $)$ were increased by FetA $(p<0.05)$. In VSMCs, Rho kinase activity was increased $(2$ fold, $\mathrm{p}<0.05)$ at $30 \mathrm{~min}$; while myosin light chain (MLC) activation was only increased (0.5 fold) at $15 \mathrm{~min}$. In summary, FetA seems to influence vascular function through Nox1-ROS dependent mechanisms, where only endothelial dysfunction and contractile responses were mediated by TLR4 activation. Identification of FetA as a ligand of TLR4 and further characterisation of FetA-induced signalling may be of importance to the development of new therapy for treatment of hypertension.

\section{AMPHIPHYSIN II (BIN1) DRIVEN TRANSVERSE TUBULE FORMATION IN CARDIAC MUSCLE}

Jessica Caldwell*, Rebecca Taylor, David Eisner, Katharine Dibb, Andrew Trafford. University of Manchester

\subsection{6/heartjnl-2017-311726.169}

Transverse (t)-tubules are vital for maintaining normal contractility of the heart through the tight regulation of excitation coupling. In cardiac disease, such as heart failure, t-tubule loss is closely associated with decreased synchrony of calcium release from the sarcoplasmic reticulum, resulting in impaired contractility. Thus, determining the mechanisms that control ttubule formation is essential for understanding cardiac disease. Evidence suggests that the protein Amphiphysin II (AmpII) controls t-tubule formation in cardiac muscle and thus, may play a vital role in calcium regulation. Several studies, including our own, have shown that gene silencing of AmpII causes t-tubule loss in both skeletal and cardiac muscle. Furthermore, in non-muscle cells that usually lack t-tubules, expression of some variants of AmpII led to tubule formation. We therefore aimed to extend these observations and determine if AmpII is sufficient to drive t-tubule formation in the heart.

Neonate rat ventricular myocytes (NRVMs) were isolated from 2 day old rats and maintained in culture. Vectors encoding isoforms 5, 8 and 9 of the AmpII gene (Bin1) with a C-terminal mKate2 fluorescent protein tag were transiently expressed in NRVMs using FuGENE 6 lipofection. A vector containing the mKate2 fluorescent tag only was used as negative control. After
48 hours, over-expression of Bin1 was confirmed at both the mRNA and protein level. Tubule formation was assessed using the membrane dye FM-464 and confocal microscopy. Of cells successfully transfected with Bin1, 95\% had developed tubule structures. Conversely, tubules were absent in cells only expressing the fluorescent tag $(\mathrm{p}<0.001)$. Furthermore, Bin 1 isoform 8 expression led to formation of more tubule structures when compared to isoform 5 and $9(\mathrm{p}<0.05)$.

To determine if Bin1 driven tubules are functional, transfected cells were loaded with the $\mathrm{Ca} 2+$ indicator Fluo-8 AM and field stimulated. When compared with untransfected myocytes, expression of Bin 1 isoforms 5,8 and 9 increased the amplitude of the systolic calcium transient $(\mathrm{p}<0.05)$. Furthermore, transfection with Bin1 isoforms 5 and 9 led to faster rise and decay of the systolic calcium transient $(\mathrm{p}<0.05)$. Transfection with the control vector only had no effect on the calcium handling when compared with untransfected cells.

Over-expression of Bin1 isoforms 5,8 and 9 led to the formation of tubular structures in NRVMs. Whilst Bin1 isoforms 8 appears to play more of a role in tubule formation in NRVMs, these data suggest that other Bin1 isoforms (5 and 9) may enhance calcium kinetics. These data therefore suggest that Bin1 plays a vital role in tubule formation and development in cardiac myocytes. Given the importance of $\mathrm{t}$ tubules to normal excitation contraction coupling and their perturbation in heart failure we therefore suggest that Bin1 might be a novel therapeutic target.

\section{$172 \beta^{2}$-CATENIN MEDIATES THE ANTI-APOPTOTIC EFFECTS OF NO IN ENDOTHELIAL CELLS}

${ }^{1}$ Virginia Tajadura Ortega ${ }^{*},{ }^{2}$ Christina Warboys, ${ }^{1}$ Albert Ferro. ${ }^{1} \mathrm{KCL} ;{ }^{2} \mathrm{CL}$

10.1136/heartjnl-2017-311726.170

$\hat{\mathrm{I}}^{2}$-Catenin Mediates the Anti-Apoptotic Effects of $\mathrm{NO}$ in Endothelial Cells

Apoptosis is implicated in a number of cardiovascular diseases. Increased endothelial cell apoptosis is associated with the development of atherosclerotic plaques. Strategies to promote endothelial cell survival may therefore represent a novel therapeutic approach in cardiovascular disease.

Nitric oxide (NO) and $\beta^{2}$-catenin have both been shown to promote cell survival. Recently we showed that pharmacological activation of the endothelial nitric oxide synthase (eNOS), acting through cGMP, can promote nuclear translocation and transcriptional activity of $\hat{\mathrm{I}}^{2}$-catenin.

Using an orbital shaker system to generate shear stress, we investigated the physiological role of $\beta^{2}$-catenin as a mediator of NO-induced cell survival in endothelial cells. Human umbilical vein endothelial cells (HUVEC) exposed to flow for 72 hour at $150 \mathrm{rpm}$ on an orbital shaker exhibited different degrees of apoptosis between the undisturbed flow (UF) zone in the periphery of the well and the central disturbed flow (DF) zone in the centre of the well $(1.54 \% \pm 0.26$ (mean \pm SEM) cleaved caspase positive cells in DF zone compared to $0.26 \% \pm 0.07$ in UF zone; $n=3 p<0.01$ ). The degree of apoptosis increased in both UF and DF areas when cells were treated with an inhibitor of beta-catenin transcriptional activity, with $4.45 \% \pm 0.86$ apoptotic cells in the DF zone compared to $0.57 \% \pm 0.11$ in the UF zone $(n=3 p<0.05$ for nontreated to inhibitor treated in the DF zone). 